\title{
ANÁLISIS NUMÉRICO DE CARACTERÍSTICAS BÁSICAS DE UNIDADES FAMILIARES PRODUCTIVAS (UFP) EN NUEVE COMUNIDADES RURALES DE NICARAGUA
}

\author{
NUMERICAL ANALYSIS OF BASIC CHARACTERISTICS OF PRODUCTIVE FAMILY UNITS \\ (UFP) IN NINE RURAL COMMUNITIES IN NICARAGUA
}

\section{Benavides-González Álvaro MSc, Morán-Centeno Juan Carlos MSc.}

Investigadores del Programa Desarrollo Participativo Integral Rural (DEPARTIR), Facultad de Agronomía, Universidad Nacional Agraria, Managua, Nicaragua, km 12 1⁄2 Carretera Norte / alvaro.benavides@una.edu.ni / Teléfono (505) 2263-2609, Ext. 377.

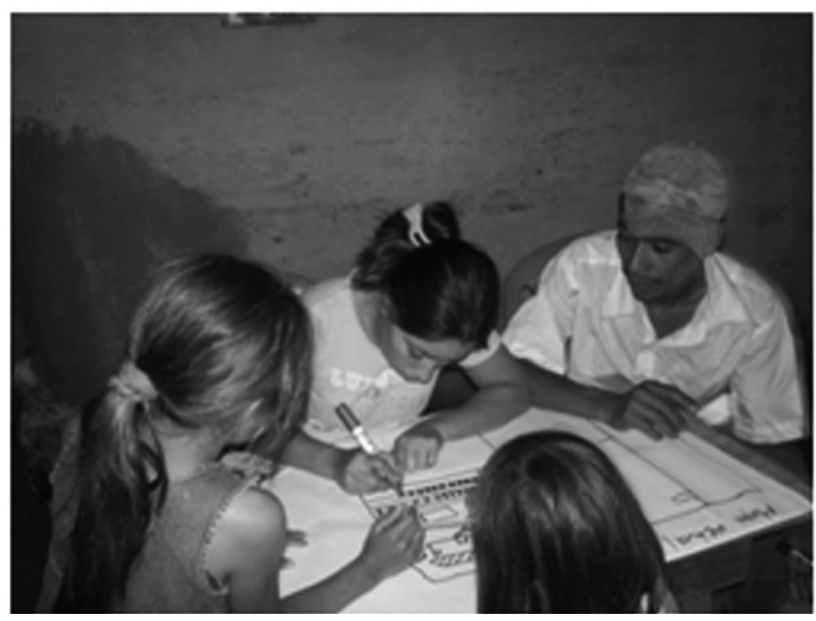

\section{RESUMEN}

El programa Desarrollo Participativo Integral Rural (DEPARTIR) interactúa con familias, estudiantes y docentes investigadores de la Universidad Nacional Agraria (UNA) en comunidades rurales de Nicaragua. Durante el período 2006-2013 el programa ha tenido intervención en 14 comunidades, desarrollando propuestas priorizadas por las familias; así como estudios de cuestionarios socieconómicos, suelos y agua, y análisis de la Unidad Familiar de Producción (UFP). Basado en la información de los municipios de Las Sabanas $(n=164)$, El Crucero $(n=207)$ y Nandaime $(n=127)$, se procedió a analizar bases de datos de 498 UFP con más de 80 variables, a través de análisis univariados y multivariados. Según las condiciones en las familias, se determinó el índice de calidad de vida en la vivienda (ICVV) y digitalizado por medio de coordenadas geográficas las nueve comunidades rurales, y en promedio mostraron un bajo nivel de vida. La religión, la tenencia de tierra, el ICVV y el sexo del informante, distanciaron las comunidades del municipio de Las Sabanas y de Nandaime. Además, el 78\% de la población en las comunidades rurales muestreada fue inferior a 40 años, y 11 personas de cada 100, no saben leer ni escribir, con una mediana del $6 \%$ y máxima de $20.7 \%$ de analfabetismo.

Palabras clave: DEPARTIR, análisis univariado y multivariado. indice de calidad de vida en la vivienda (ICVV), analfabetismo.

\begin{abstract}
The program Desarrollo Participativo Integral Rural (DEPARTIR) interacts with families, students and researchers of the Universidad Nacional Agraria (UNA) in rural communities of Nicaragua. During the period 2006-2013 the program has had involvement in 14 communities prioritized proposals developed by families, as well as socioeconomic questionnaires studies, analysis of soil and water, and analysis of Productive Family Units (UFP). Based on information from the municipalities of Las Sabanas $(n=164)$, El Crucero $(n=207)$ and Nandaime $(n=127)$, proceeded analyze databases of 498 UFP over 80 variables through univariate analysis and multivariate. Depending on the conditions in families, we determined the quality of life in the dwelling (ICVV) and digitized using geographic coordinates in nine rural communities, and on average showed a low standard of living. The religion, land tenure, the ICVV and sex of the informant, distanced communities in the municipality of Las Sabanas and Nandaime. In addition, $78 \%$ of the population in rural communities sampled was less than 40 years, and 11 people out of 100 , not can read or write, with a median of $6 \%$ and a maximum of $20.7 \%$ illiteracy.
\end{abstract}

Key words: DEPARTIR, univariate and multivariate analysis, quality of life in the dwelling (ICVV), illiteracy. 
$\mathrm{E}$ 1 programa de Desarrollo Participativo Integral Rural (DEPARTIR) ofrece propuestas concretas para apoyar el progreso sostenible en áreas rurales. Desde el año 2006, ha desarrollado seis diagnóstico participativos en 14 comunidades rurales de Nicaragua, en los municipios de Las Sabanas, Granada, Chinandega-Villanueva y El Crucero. En estos Diagnósticos participan e interactúan familias de las comunidades, estudiantes y docentes investigadores de la Universidad Nacional Agraria (Querol et al., 2012). La información abarca estudios de cuestionarios socieconómicos, suelos y agua; así como el análisis de la Unidad Familiar de Producción (UFP).

Mediante la información recabada, se hacen análisis exploratorios y desarrollan índices para relacionar comunidades, tales como el índice de calidad de vida en la vivienda (Salas-Borgoin, 2012), índice de masculinidad (INIDE, 2007) y tasa de analfabetismo COVEG (2013), entre otros.

Por lo anterior, el presente estudio tiene como finalidad relacionar variables e índices de comunidades rurales de Nicaragua mediante digitalización geográfica y herramientas estadísticas univariadas y multivariadas.

\section{MATERIALES Y MÉTODOS}

La información recopilada en cuestionarios por estudiantes participantes de las facultades de la UNA, fue analizada mediante herramientas de análisis multivariados y aspectos básicos de Sistemas de Información Geográfica (SIG), por lo que el estudio abarca los aspectos cualitativos y cuantitativos.

En la figura 1, se aprecian los puntos de intervención de DEPARTIR. Con una base de datos de más 800 registros de las familias (UFP), e información principal de variables de género, edad, ocupación principal, nivel académico y alfabetismo superior a 3000 registros.

Para el cálculo de la muestra de datos socieconómicos, se utilizó la ecuación propuesta por Aguilar-Barojas (2005) en poblaciones finitas y variables categóricas.

$$
\mathrm{n}=\frac{N Z^{2} p q}{d^{2}(N-1)+Z^{2} p q}
$$

Donde:

$\mathrm{n}=$ es el tamaño de la muestra

$\mathrm{N}=$ tamaño de la poblacional

$\mathrm{Z}=$ valor de $\mathrm{Z}(\infty=0.05, \mathrm{Z}=1.96)$

$\mathrm{p}=$ proporción aproximada del fenómeno en

estudio de la población

$\mathrm{q}$ = proporción de la población de referencia que no

representa el fenómeno (1-p)

$\mathrm{E}=\mathrm{d}=$ Porcentaje de error asumido

Con la ecuación anterior se obtuvo una muestra de 498 encuestas, en las que se incluyeron comunidades distribuidas de la siguiente manera:

Las Sabanas (Madriz, 2011): Buena Vista (103), El Pegador (31), Nueva Esperanza (30)
El Crucero (Managua, 2012): Las Pilas 2 (82), Las Pilas 1 (75), El Callao (50)

Nandaime (Granada, 2013): Nandarola (88), San Mateo (29), Los Castillos (10)

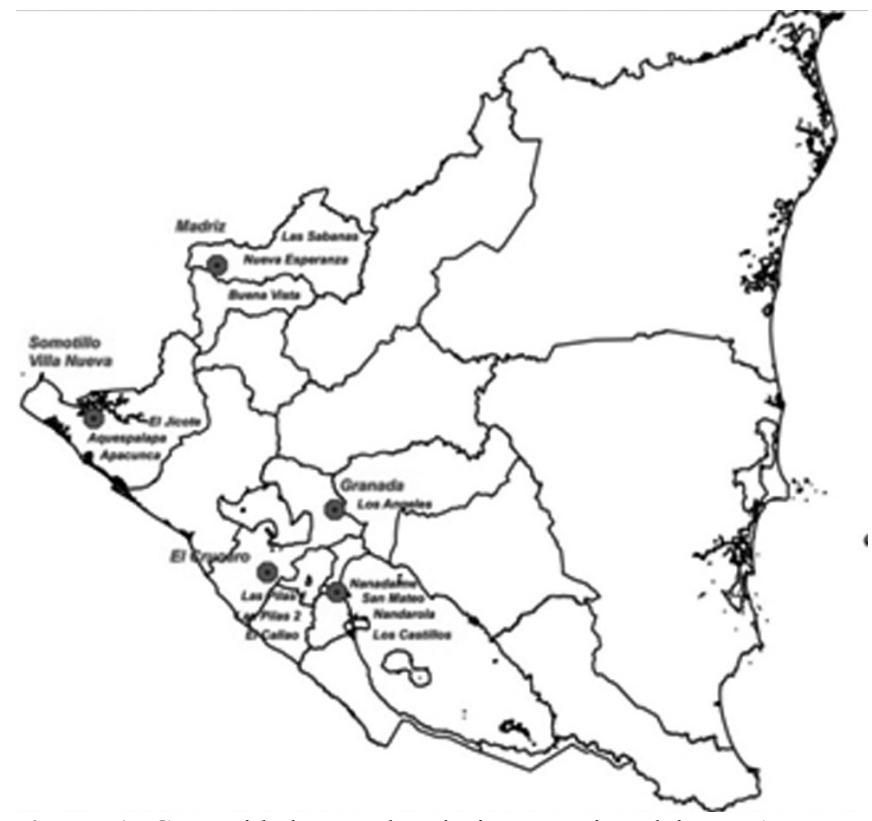

Figura 1. Comunidades rurales de intervención del DEPARTIR, período 2006-2013.

\section{Variables evaluadas}

Variables discriminatorias. A través de la selección en más de 80 variables, se trabajó con área de la UFP en manzanas (X1), número de miembros en la casa (X2), sexo (X3), edad (X4), tenencia de la tierra (X5), tipo de organización (X6), tipo de religión $(\mathrm{X} 7)$, meses de acceso a la carretera principal (X8), producción agrícola (X9), e indice calidad de vida de la vivienda (ICVV) por medio de variables de las condiciones del hogar de cada familia (UFP).

Índice calidad de vida de la vivienda (ICVV). Para la determinación del índice de calidad de vida de la Vivienda (ICVV), se utilizó la metodología propuesta por SalasBorgoin (2012), asumiendo una escala del cero al 10 en las categorías de las variables, considerando al cero como el valor más bajo o despreciable, y el 10 para el valor superior o mejor condición.

Los componentes calculados del ICVV fueron: condiciones sanitarias (CS) conformado por el tipo de letrina; condiciones físicas de la vivienda (CFV) integrado por el material predominante de la pared, piso, techo y estado de la vivienda; ambiente en la casa (AC) que integra al número de cuartos; servicios básicos (SB) como el servicio de energía y 
combustible para cocinar. Cada una de estas condiciones se promedió para cada caso: $\mathrm{ICVV}=\mathrm{CS}(0.3)+\mathrm{CFV}(0.3)+$ $\mathrm{AC}(0.2)+\mathrm{SB}(0.2)$.

Variables dicotómicas y cuantitativas. Variables ordinales, nominales y discretas fueron transformadas a doble estados, éstas fueron X3 (hombre, mujer), X5 (si y no propietario), X6 (si y no organizado), X7 ( tiene o no religión), X9 (si o no consumo) para combinarlas con variables continuas $\mathrm{X} 1$, $\mathrm{X} 2, \mathrm{X} 4$ y X8.

Pirámide poblacional, índice de masculinidad y tasa de analfabetismo. Los rangos de la pirámide de población de las comunidades, índice de masculinidad y tasa de analfabetismo se obtuvieron mediante la metodología de INIDE (2007), COVEG (2013).

$$
I M=\frac{P M}{P F} 100
$$

Donde:

IM: Número de hombres por cada 100

mujeres en la localidad

PM y PF, es la población masculina y femenina

en la comunidad, respectivamente

$$
\text { Analfge }=\frac{P_{g e}^{\text {Analf }}}{P_{g e}} 100
$$

Donde:

Analfge: Porcentaje de la población analfabeta en el grupo de edad ge en la comunidad.

ge: son los grupos de edad: $15-24,25-34,35-44,45-54,55-$ 64 , y más de 15 años.

$P g e^{\text {Analf }}$ : Número de personas analfabetas en el grupo de edad ge.

Pge: es la población total en el grupo de edad ge.

Análisis estadístico. Las variables fueron procesadas en hojas electrónicas y analizadas con InfoStat v. 2012 y SPSS v. 19. Se utilizaron estadísticos descripticos, correlaciones, análisis univariados: Kruskal-Wallis, ANOVA, LSD; análisis multivariados: Análisis de Correspondencia (ANARE), Análisis de Componentes Principales (ACP) y Análisis de Coordenadas Principales (EMD).
El manejo de variables consistió en estandarización de variables en ACP (1/S), distancia de Gower (1/S-1) en EMD, Ward y Gower en AA, test de Kruskal-Wallis en ICVV; además de digitalización de coordenadas geográficas con ArcGIS (v. 10).

\section{RESULTADOS Y DISCUSIÓN}

El problema de la pobreza en Nicaragua impide el pleno disfrute de los derechos humanos, en especial los económicos, sociales y culturales. La asignación de los recursos al gasto social es muy escasa en relación con la demanda de la población, poniendo en riesgo el acceso y la calidad de los servicios básicos fundamentales que requieren las personas para vivir en condiciones mínimas de satisfacción, por ejemplo: el acceso a salud, educación, empleo digno, vivienda, agua y saneamiento, entre otros (CCPDH, 2008).

Índice calidad de vida de la vivienda (ICVV). El ICVV, se fundamenta en un procedimiento sencillo con principios básicos del análisis multicriterio, dirigido a seleccionar, del conjunto de características que tiene una vivienda y que registran los censos de un país, las fundamentales para garantizar bienestar en la población y, a partir de ello, jerarquizarlas y evaluarlas a través de diversos criterios o condiciones (Salas-Borgoin, 2012).

Al ICVV se aplicó el test de Kruskal-Wallis (H), basado en la suma de los rangos asignados a las observaciones dentro de cada muestra (Sidney, 1983), en este caso comunidad. La metodología utilizada sobre las agrupaciones múltiples son descritas por Conover (1999); citado por Rienzo et al., (2008).

En el tabla 1, se aprecian los promedios de los componentes utilizados con sus respectivas agrupaciones propias de los rangos calculados. La significación estadística fue alta $(\infty<0.001)$, ya que los valores de $\mathrm{H}$ calculados superaron a $\mathrm{X}^{2}$.

Condiciones sanitarias (CS). En las nueve comunidades muestreadas, el $55.2 \%$ de las familias tuvieron el servicio de eliminación de excretas, y la población restante no (44.8\%). Estos datos no contrastan con la media nacional expuesta por FIDEG (2009), en la que asevera que sólo el $22.1 \%$ de las comunidades rurales tienen este servicio inadecuado. 
Tabla 1. Significación estadística con el test de Kruskal-Wallis sobre variables de condiciones de la vivienda en nueve comunidades rurales de Nicaragua

\begin{tabular}{|c|c|c|c|c|c|c|c|c|c|c|}
\hline \multirow{2}{*}{$\begin{array}{l}\text { Comunidad } \\
\text { San Mateo }\end{array}$} & \multicolumn{2}{|l|}{ CS } & \multicolumn{2}{|c|}{ CFV } & \multicolumn{2}{|l|}{$\mathbf{A V}$} & \multicolumn{2}{|l|}{ SB } & \multicolumn{2}{|c|}{ ICVV } \\
\hline & 1.30 & $\mathrm{~b}$ & 1.56 & $\mathrm{a}$ & 0.32 & $\mathrm{a}$ & 0.19 & $\mathrm{a}$ & 3.37 & $\mathrm{a}$ \\
\hline Las Pilas 1 & 0.20 & $\mathrm{a}$ & 1.84 & $\mathrm{bc}$ & 0.42 & $\mathrm{c}$ & 1.27 & $\mathrm{c}$ & 3.72 & $\mathrm{~b}$ \\
\hline Las Pilas 2 & 0.16 & $\mathrm{a}$ & 1.91 & $\mathrm{c}$ & 0.35 & $a b$ & 1.38 & $a b$ & 3.81 & $\mathrm{~b}$ \\
\hline El Callao & 0.13 & $\mathrm{a}$ & 1.77 & $\mathrm{bc}$ & 0.49 & $\mathrm{~cd}$ & 1.68 & $\mathrm{~cd}$ & 4.07 & $\mathrm{a}$ \\
\hline Buena Vista & 1.41 & $\mathrm{~b}$ & 1.57 & $\mathrm{a}$ & 0.40 & $\mathrm{bc}$ & 1.23 & $\mathrm{bc}$ & 4.61 & $\mathrm{c}$ \\
\hline Nandarola & 1.20 & $\mathrm{~b}$ & 1.56 & $\mathrm{a}$ & 0.33 & $\mathrm{a}$ & 1.66 & a & 4.75 & $\mathrm{~b}$ \\
\hline Los Castillos & 1.56 & $\mathrm{~b}$ & 1.68 & $a b$ & 0.38 & $a b c$ & 1.80 & $a b c$ & 5.42 & d \\
\hline Nueva Esperanza & 1.40 & $\mathrm{~b}$ & 1.74 & $\mathrm{~b}$ & 0.45 & $\mathrm{c}$ & 1.93 & $\mathrm{c}$ & 5.53 & d \\
\hline El Pegador & 1.50 & $\mathrm{~b}$ & 1.79 & bc & 0.54 & d & 1.81 & d & 5.64 & d \\
\hline $\mathrm{H}$ & 206.06 & & 88.58 & & 44.29 & & 142.79 & & 162.79 & \\
\hline $\operatorname{Pr}$ & $<0.015$ & & $<0.001$ & & $<0.001$ & & $<0.002$ & & $<0.001$ & \\
\hline
\end{tabular}

$\overline{\mathrm{CS}}=$ Condiciones sanitarias, $\mathrm{CFV}=$ Condiciones físicas de la vivienda, $\mathrm{AV}=\mathrm{Ambiente}$ de la vivienda $\mathrm{SB}=$ Servicios básicos, ICVV = Índice de calidad de vida en la vivienda. Medias con igual letra, no difieren $(\infty=0.05)$.

Condiciones físicas de la vivienda (CFV). Viviendas de Las Sabanas, El Crucero y Nandaime, presentaron techo de zinc $(87.1 \%)$, tejas $(9.2 \%)$, y el restante de palma, nicalit, madera, entre otros. El piso de tierra $(65.9 \%)$, concreto $(21.7 \%)$, ladrillo $(11.6 \%)$, y otros $(0.8 \%)$. El material más utilizado en las paredes fueron: madera $(43.6 \%)$, adobe $(21.3 \%)$, bloque (18.1), ladrillo (8\%), cantera $(2.6 \%)$, zinc $(2 \%)$, bambú $(1.6 \%)$, cartón $(0.2 \%)$, entre otros.

Según FIDEG (2009), la proporción de hogares con materiales en la vivienda inadecuados en la población rural es del $64 \%$ en piso, $6.3 \%$ en paredes y $3.6 \%$ en techo. Las condiciones físicas de las viviendas (CFV) en las comunidades muestreadas (tabla 1), contrastan con estas características.

Ambiente de la vivienda (AV). Las comunidades de los municipios muestreados, en su mayoría las UFP tenían entre uno y dos cuartos en la habitación (73.9\%). Se encontraron 197 casos con un cuarto (39.6\%), 171 con dos cuartos $(34.3 \%)$, 80 con tres cuartos (16.1\%), 40 con cuatro cuartos $(8 \%)$, y 10 hogares $(2 \%)$ presentaron entre cinco y siete cuartos.

Servicios básicos (SB). Un componente de servicio básico en los hogares es el servicio de energía eléctrica y el abastecimiento de agua. Algunas de las comunidades muestreadas no contaron con este servicio (24.5\%), por lo que tienen que recurrir a alumbrarse con candil y/o candela. El uso de otras fuentes de energía alternativas como es el caso de energía solar fue muy bajo (14.5\%), a excepción de la comunidad de Buena Vista (Las Sabanas) que presentó el 96\% de paneles solares.
El abastecimiento de agua fue del $59.6 \%$, y un $39.4 \%$ no contó con el servicio de agua potable en las nueve comunidades estudiadas. FIDEG (2009), reporta que el $26.1 \%$ de la población rural no tiene este servicio. Cabe señalar que algunas familias de las comunidades rurales muestreadas consumen agua de pozo, manantiales, pozos comunales o agua de río, entre otros.

Estudios realizado por la UCA (2010), indican que el $22 \%$ de la población nicaragüense obtienen el servicio de agua potable por tuberías. El INEC (1995), menciona que el $39 \%$ de la población nicaragüense del área rural cuenta con este servicio ya sea por medio de acueductos, comité de agua potable en zonas rurales, y puestos públicos (pozos con bombeo manual). Generalmente la provisión de agua tiene como fuente de captación las aguas subterráneas. Esto incide en el suministro en la estación seca, donde en algunas zonas la población no dispone de agua en algunos días de la semana, tal es el caso de las comunidades de Las Pilas 1, Las Pilas 2 y El Callao.

FIDEG (2009), afirma que en la zona rural el $47 \%$ adolece de energía eléctrica; por otro lado, la UCA (2010), reporta que en Nicaragua el $68 \%$ de los hogares no cuenta con energía eléctrica, siendo las comunidades más alejadas del país las que no cuentan con este servicio básico.

A continuación se presentan las dimensiones que componen al ICVV en las comunidades estudiadas.

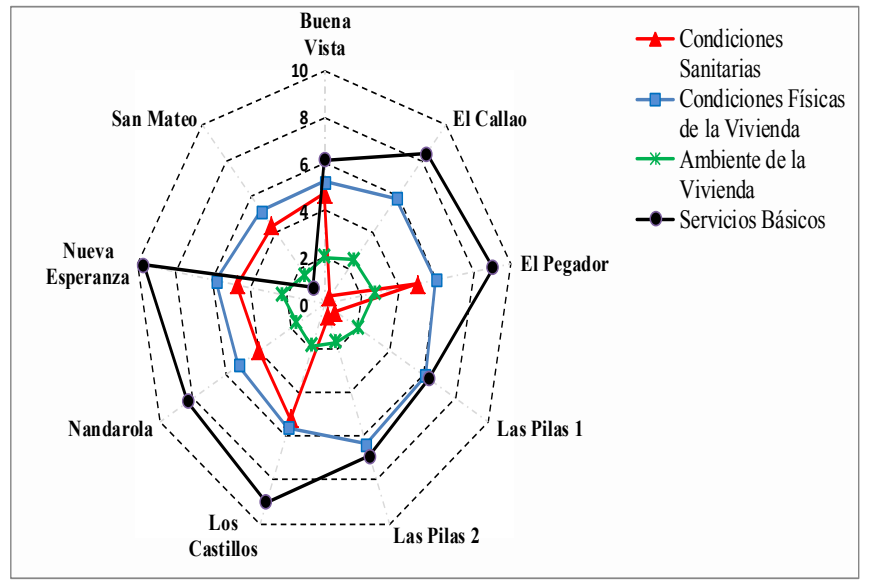

Figura 2. Relación de componentes básicos en la vivienda de nueve comunidades rurales de Nicaragua. 
En Nicaragua, $67 \%$ de la población rural está en la pobreza; y de esto el $49.6 \%$ de las familias están en la pobreza no extrema, y $18.2 \%$ se ubica en pobreza extrema (FIDEG, 2009).

A través de la figura 2 , se deduce que las comunidades muestreadas están dentro del rango de pobreza general, aunque en algunas comunidades estudiadas se ubicaron en la extrema pobreza (tabla 1).

Salas-Borgoin (2012), calculó las dimensiones para estimar ICVV y obtuvo los siguientes valores: CS (1.35), CFV (1.62), SB (0.93), SB (1.24) y un valor de 5.14 para el ICVV. Los valores fueron clasificados en la categoría de moderada calidad de vida en la vivienda.

Análisis de correspondencia (ANARE). El ANARE, es una técnica de interdependencia que sirve para reducir dimensiones y conformar congregación, y su aplicación más directa es la de graficar una correspondencia de categorías de variables, en particular las nominales (Hair et al., 2005; citado por Raimundini et al., 2009).

En la figura 3, se presentan los ICVV mediante coordenadas geográficas y comparadas con ANARE relacionando comunidades. Los rangos escogidos fueron los de mayor valor de Cronbach (0.95) y puntos de inercia $(0.68,0.21)$, lo que representó una mejor apreciación de las categorías asociadas.

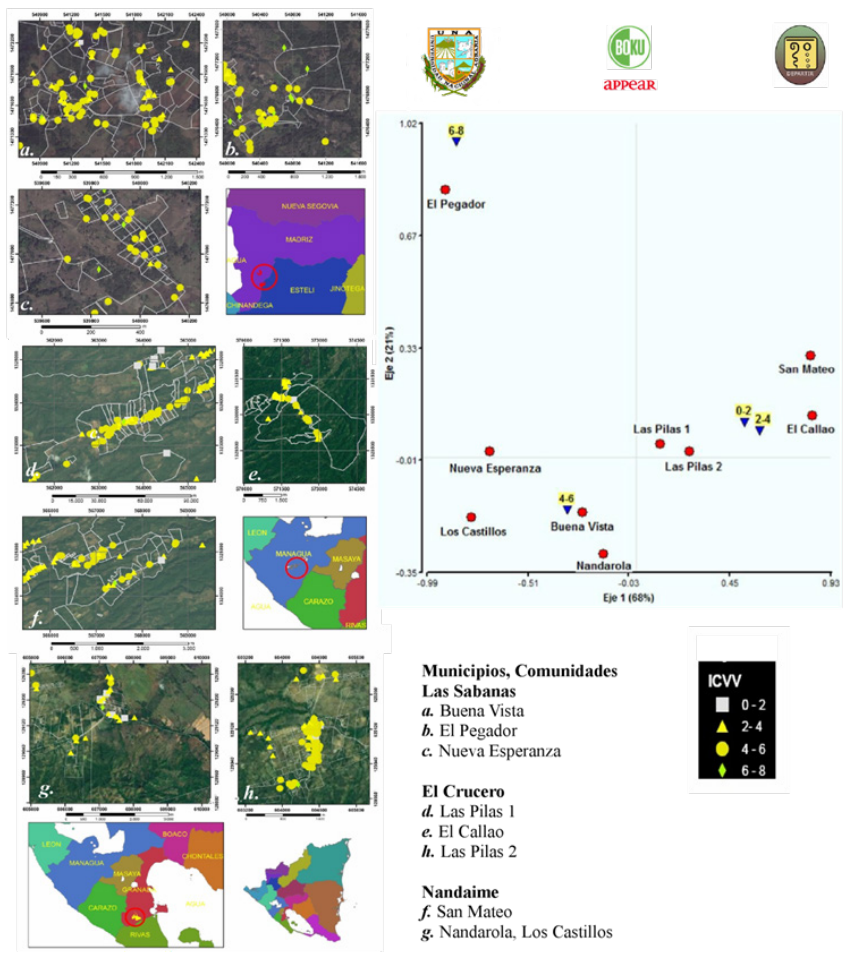

Figura 3. Digitalización geográfica del ICVV y análisis de correspondencia de nueve comunidades en Las Sabanas, El Crucero y Nandaime.
En el plano cartesiano de la figura 3, se observan los dos ejes de inercia, en el cual el primer eje explica el $68 \%$ de la variación total y agrupó a las comunidades de San Mateo, El Callao, Las Pilas 1 y Las Pilas 2, con la valoración de ICVV entre 0-2 y 2-4. Por otro lado, las comunidades de Nueva Esperanza, Los Castillos, Buena Vista y Nandarola comparten un mejor nivel de condiciones con un ICVV de 4-6. El Pegador entre 6-8. Esto obedece a que la mayoría de la casas de El Pegador son producto de un proyecto de financiamiento post huracán Mitch, lo que hace que esta comunidad difiera de las otras. Similar explicación de agrupación es sugerida para el eje 2. El 89\% en los ejes de inercia hace indicar una considerable explicación de variación del análisis, por lo que esta aseveración es apoyada por el estadístico alpha de Cronbach como coeficiente de mayor fiabilidad (Ledesman et al., 2002).

Pirámide poblacional en las comunidades. La población de las comunidades fueron agrupadas en tres categorías, el $33 \%$ estuvo representada por jóvenes menores a 14 años, $62 \%$ adultos entre 15 y 64 años, y personas de la tercera edad superior a los 65 años. En la figura 4, se observa el engrosamiento de la pirámide progresiva, conforme disminuyen los rangos en las edades de las personas. Esto es propio de una población en aumento y con alta tasa de crecimiento, en la que sobresalen los rangos de edades entre 0-24 años. Se encontró que el $76.3 \%$ de los comunitarios tienen edades inferiores a los 40 años.

La población menor a 15 años para el año 2002, según CELADE (2004) fue de $44.1 \%$ en Guatemala, $35.6 \%$ en El Salvador, $41.6 \%$ en Honduras, $42 \%$ en Nicaragua, $31.8 \%$ en Costa Rica y $31.9 \%$ en Panamá. En el caso de América Latina el promedio general fue de $31.9 \%$ de individuos con edades inferiores a los 15 años. El PNUD (2007), reporta que en el 2005 , el $37.9 \%$ y el $4 \%$ correspondió a personas menores a 15 años y mayores a los 65 años, respectivamente.

La población menor a los 15 años fue del 35.4\% (El Crucero 14.1\%, Granada 8.2\%, Las Sabanas 13.1\%), y los estadígrafos básicos fueron similares en las comunidades, con una media de 26.44 años y una desviación típica de 19.30 años, lo que indica una población relativamente joven.

Esta población estudiada es joven y está en desequilibrio con los puestos de trabajo, emigraciones, aumento de algunos servicios, entre otras. Los resultados coinciden con la situación nacional, en la que según el INIDE (2007), Nicaragua es un país que se encuentra en una etapa intermedia de la transición demográfica, con una fecundidad moderada y baja mortalidad. Esta particularidad se resume en una población con crecimiento moderado y con una estructura por edades de una población más bien joven. A medida que se avance en el proceso de transición demográfica, se tendrá un menor crecimiento y una estructura más envejecida. No se puede dejar de mencionar que el proceso migratorio observado en Nicaragua acelera este proceso. 


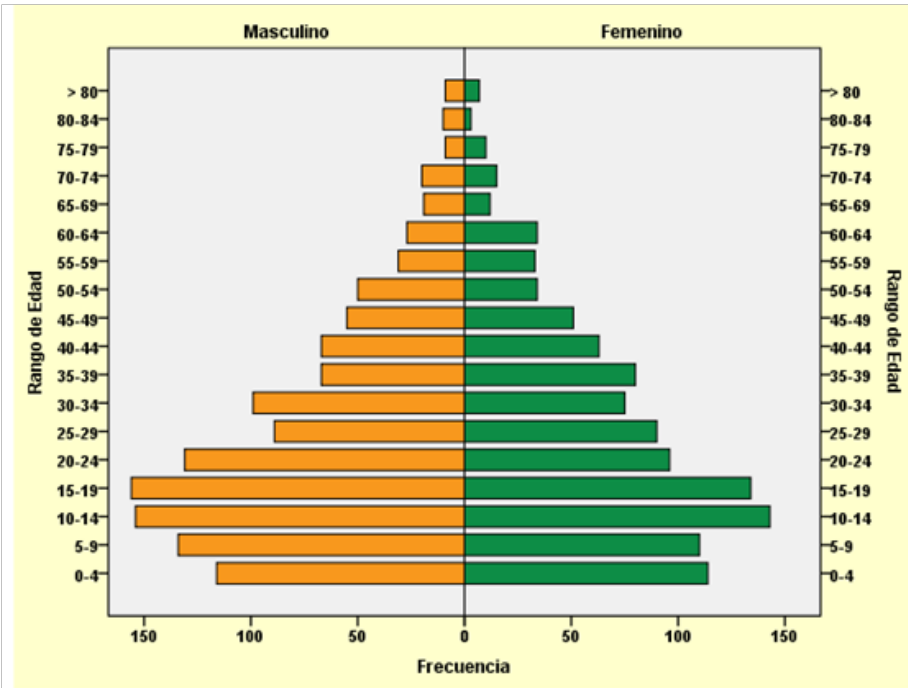

Figura 4. Pirámide poblacional según el sexo en nueve comunidades rurales de Nicaragua. $\mathrm{N}=2347$.

Índice de masculinidad en las comunidades. El índice de masculinidad, según CELADE (2004), fue en 2002 del 96.5\% en Guatemala, 96.4\% en El Salvador, 101.6\% en Honduras, $99.7 \%$ en Nicaragua, $103.5 \%$ en Costa Rica y $98.7 \%$ en Panamá. En el caso de América Latina el promedio general fue de 98.4\%. Asimismo, el INIDE (2007), reportó índice de masculinidad nacional del 97.2\% para el año 2005 (figura 5).

En las comunidades rurales muestreadas y agrupadas en municipios, la población de El Crucero, presentó los mayores valores promedios de índice de masculinidad, y en el caso de los municipios de Granada y Las Sabanas el número de mujeres en relación al número de hombres fue mayor. Asimismo, el índice de masculinidad se mantuvo uniforme en las comunidades muestreadas de los tres municipios, con menor variación en las edades de los municipios de Granada y Las Sabanas (figura 5). Los índices promedios calculados fueron de 120.5, 43.18 y 51.71, para El Crucero (El Callao, Las Pilas 1, Las Pilas 2), Granada (San Mateo, Nandarola, Los Castillos) y Las Sabanas (Nueva Esperanza, El Pegador, Buena Vista).

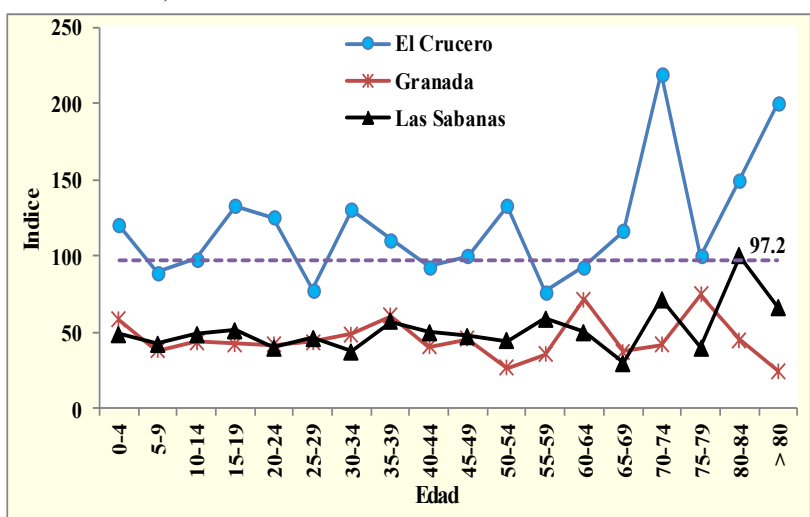

Figura 5. Índice de masculinidad por edades en tres municipios rurales de Nicaragua. $\mathrm{N}=2347$.
COVEG (2013), menciona que una población mantiene una relación natural de 97 varones por cada 100 mujeres, y este debería de ser el índice de masculinidad natural de una población, no obstante por razones sociales puede ser superior o inferior por regiones mundiales, nacionales o subnacionales.

Al comparar el índice de masculinidad del año 2004, sólo las comunidades del municipio de El Crucero corresponde con el 97.2\% reportado por el INIDE (2007), en cambio las comunidades de los municipios de Granada y El Crucero se muestrearon más mujeres que hombres.

En el caso de las comunidades San Mateo, Nandarola y Los Castillos (Granada), muchos de los hombres trabajan por temporada agrícola en Costa Rica. De igual manera, algunos hombres de las comunidades El Pegador, Nueva Esperanza y Buena Vista (Las Sabanas), van a trabajar a El Salvador y Honduras. Lo anterior, contrasta con lo expuesto por el INIDE (2007), al indicar que el índice de masculinidad en los censos es similar y asume valores inferiores a 100 en edades tempranas, por lo que, entre otras cosas, indica una estructura de migración por sexo y edad similar en las últimas décadas.

Tasa de analfabetismo. La educación es determinante para potenciar el desarrollo integral de un país. El capital humano acumulado, es lo que permite mejorar las condiciones de vida de la población. A mayor nivel de educación y formación se traducen en mayores y mejores oportunidades de empleo y condiciones de vida (INIDE, 2005). Por otro lado, la UCA (2010), reporta que los pobres extremos no alcanzan un buen nivel educativo, ya que el $41 \%$ no tiene ningún nivel de instrucción y el $50.6 \%$ únicamente logra cursar la primaria.

Lestage (1982), asevera que existe una relación del analfabetismo con la demografía, y la relación es importante no sólo por las consecuencias cuantitativas, sino también en el plano cualitativo, ya que la multiplicidad y la complejidad de los factores que intervienen en los fenómenos demográficos pueden incidir en los programas de alfabetización, y asevera que el analfabetismo es inherente al grado de subdesarrollo de un país. Sobre esto, el MECD (2004) agrega que la educación es un factor primordial estratégico, prioritario y condición indispensable para el desarrollo social y económico de Nicaragua.

La prueba de Fisher (LSD), determinó que la tasa promedio de analfabetismo de El Callao, Las Pilas 1 y Nandarola fue similar $(\mathrm{Pr}=0.01)$. La tabla 2, muestra la categorización estadística de las comunidades estudiadas.

En las nueve comunidades estudiadas, el total de analfabetos mayores de 15 años fue del 52\% (hombres) y $48 \%$ (mujeres). Las comunidades de Buena Vista y Las Pilas 1, presentaron los mayores porcentajes de analfabetismo, con $13 \%$ y $10.4 \%, 10.9 \%$ y $10.4 \%$ para hombres y mujeres, respectivamente. 
La tasa de analfabetismo para América Latina y el Caribe en las edades mayores a 15 años, fue del $12.7 \%$ para el año 2000 (REDIE, 2000; citado por DGCyE, 2000).

Según PNUD (2007), la tasa de analfabetismo durante el período 1995-2005, fue del 30.9\% en Guatemala, 19.4\% en El Salvador, $20 \%$ en Honduras, $23.3 \%$ en Nicaragua, $5.1 \%$ en Costa Rica y $8.1 \%$ en Panamá, con una media general del 9.7\% de analfabetismo en América Latina y el Caribe.

Con datos preliminares, el MINED (2009); citado por CODENI, informa de un $22.2 \%, 20.2 \%$ y $7.5 \%$ de analfabetismo para los años 2006, 2007 y 2008, respectivamente. Por otro lado, aepcfa-girona (2013), considera que Nicaragua ya situó el índice de analfabetismo por debajo del 5\%, y que está a punto de conseguir el acceso universal de la infancia a la educación fundamental e implementar medidas eficaces para evitar el analfabetismo de retorno y erradicar el analfabetismo político.

Aunque la tasa promedio general de analfabetismo en las comunidades estudiadas para las personas mayores de 15 años fue de $10.65 \%$, en algunas comunidades como Las Pilas 1, Buena Vista y El Callao, los valores fueron de $19.9 \%, 14.29 \%$ y $13.92 \%$, respectivamente, también otras comunidades presentaron niveles de analfabetismo bajos (tabla 2). Se observó que las tasas promedios de analfabetismo fueron mayores a medida que la edad incrementaba (Pearson=0.81, $\mathrm{P}=0.001$ ); asimismo el analfabetismo con los rangos de edades presentaron correspondencia significativa (Kendall $=0.90, \mathrm{P}=0.001$ ). métrica, el cual se auxilia de una metodología de agrupación y una distancia que realiza una clasificación (Cuadras, 2012).

$\mathrm{Al}$ integrar el ICVV con las variables discriminantes y realizar el ACP, los tres componentes principales extrajeron el $73 \%$ de la desviación total, con $37 \%, 20 \%$ y $17 \%$, respectivamente. El grado de correlación de las variables con respecto a los componentes principales es inherente al aporte de las variancias a los mismos (tabla 3). Análogo al ACP, el EMD aisló variación de $57 \%, 15 \%$ y $10 \%$ de los primeros componentes, con $82 \%$ de varianza total. El AA, al igual que el ACP relacionó con un $\mathrm{CC}=85$, las comunidades mediante la jerarquía de Ward y Gower.

Tabla 3. Correlación y aporte de las variables en los tres primeros componentes principales. $\lambda 1=3.67, \lambda 2=1.98$ y $\lambda 3=1.78$. $\mathrm{CC}=0.99$

\begin{tabular}{ccccccc}
\hline Variable & \multicolumn{3}{c}{ Correlación (r) } & \multicolumn{3}{c}{ Aporte (\%) } \\
\hline & CP 1 & CP 2 & CP 3 & CP 1 & CP 2 & CP 3 \\
\hline X1 & 0.12 & 0.87 & 0.19 & 0.36 & 37.21 & 1.96 \\
X2 & 0.34 & -0.46 & -0.15 & 3.24 & 10.24 & 1.44 \\
X3 & 0.68 & 0.08 & 0.63 & 12.25 & 0.36 & 23.04 \\
X4 & -0.14 & -0.46 & 0.83 & 1.44 & 10.89 & 40.96 \\
X5 & 0.87 & -0.06 & -0.36 & 21.16 & 0.09 & 7.84 \\
X6 & -0.35 & -0.40 & -0.54 & 1.69 & 7.84 & 17.64 \\
X7 & 0.90 & -0.36 & 0.17 & 22.09 & 6.76 & 1.69 \\
X8 & 0.60 & 0.60 & -0.14 & 9.61 & 18.49 & 1.21 \\
X9 & 0.54 & -0.38 & -0.02 & 7.84 & 7.29 & 0.09 \\
\hline ICVV & 0.85 & 0.02 & -0.25 & 20.25 & 0.81 & 4.00 \\
\hline
\end{tabular}

Tabla 2. Significación y tasa de analfabetismo en diferentes rangos de edades en nueve comunidades rurales de Nicaragua

\begin{tabular}{lcccccccccc}
\hline & $15-24$ & $25-34$ & $35-44$ & $45-54$ & $55-64$ & $25-64$ & $>15$ años & LSD \\
\hline El Callao & 1.67 & 4.08 & 30.43 & 27.27 & 41.67 & 17.89 & 13.92 & 19.50 & $\mathrm{a}$ \\
Las Pilas 1 & 8.77 & 8.77 & 10.53 & 25.00 & 33.33 & 17.19 & 19.90 & 17.64 & $\mathrm{a}$ \\
Nandarola & 8.75 & 7.41 & 9.80 & 17.86 & 42.86 & 15.03 & 13.87 & 16.51 & $\mathrm{a}$ \\
Buena Vista & 7.94 & 10.00 & 2.08 & 18.18 & 26.09 & 11.89 & 14.29 & 12.92 & $\mathrm{ab}$ \\
Las Pilas 2 & 1.09 & 3.51 & 10.14 & 7.50 & 39.13 & 11.11 & 11.62 & 12.01 & $\mathrm{ac}$ \\
El Pegador & 5.41 & 0.00 & 0.00 & 6.25 & 28.57 & 4.84 & 5.94 & 7.29 & $\mathrm{bd}$ \\
San Mateo & 5.00 & 0.00 & 5.26 & 0.00 & 22.22 & 5.36 & 5.62 & 6.21 & $\mathrm{bd}$ \\
Nueva Esperanza & 0.00 & 5.88 & 4.00 & 8.33 & 0.00 & 5.08 & 4.21 & 3.93 & $\mathrm{~cd}$ \\
Los Castillos & 0.00 & 0.00 & 0.00 & 0.00 & 0.00 & 0.00 & 6.45 & 0.92 & $\mathrm{~d}$ \\
\hline \multicolumn{1}{c}{ LSD } & $4.29 \mathrm{~b}$ & $4.41 \mathrm{~b}$ & $8.03 \mathrm{~b}$ & $12.27 \mathrm{~b}$ & $25.99 \mathrm{~b}$ & $9.82 \mathrm{~b}$ & $10.65 \mathrm{~b}$ &
\end{tabular}

Medias con igual letra, no difieren $(\infty=0.05)$

Análisis de componentes principales (ACP) y análisis de Agrupamiento (AA). Los objetivos del ACP, es generar nuevas variables que expresen la información original, reducir la dimensión, y descartar variables correlacionadas y de menor inferencia en la variación (Pla, 1986). En cambio la representación del AA, está basado en una jerarquía indexada y ulta-
La religión (X7), la tenencia de la tierra (X5), el ICVV y el sexo (X3) caracterizaron a las comunidades con el 37\% de desviación, y congregaron comunidades de Las Sabanas y la comunidad de Los Castillos (Nandaime), y El Crucero con comunidades de Nandaime (Nandarola y San Mateo). El análisis de conglomerados confirman esta situación (figura 6). 


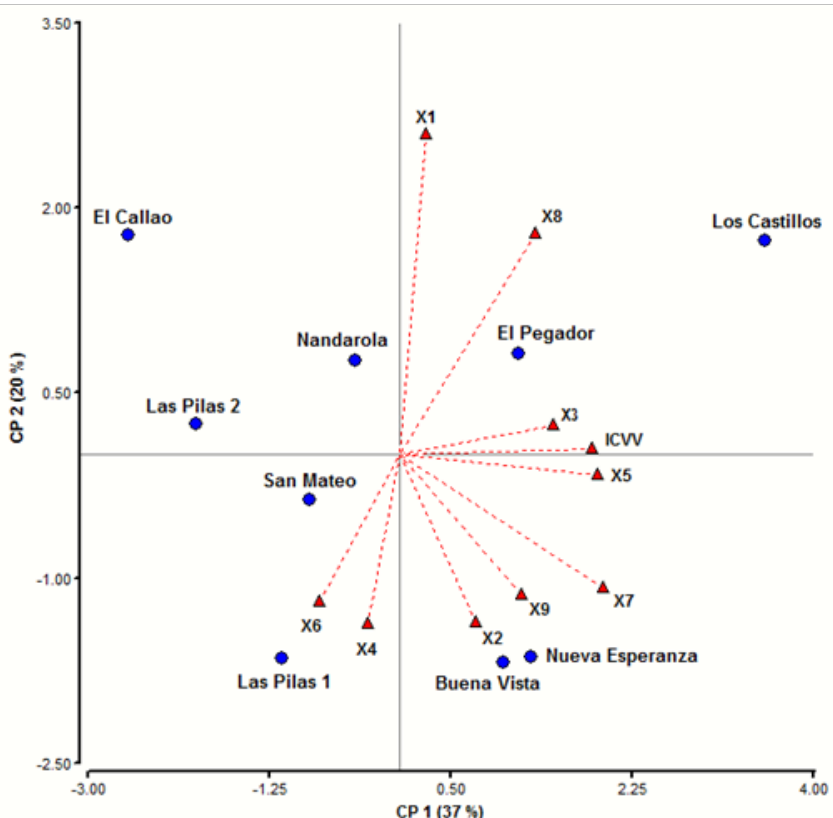

Figura 6. Análisis de componentes principales en nueve comunidades rurales de Nicaragua. $\mathrm{CC}=0.87$.

\section{CONCLUSIONES}

Mediante la implementación del índice de calidad de vida en la vivienda (ICVV) en las nueve comunidades rurales muestreadas se demostró la pobreza en las familias de las unidades familiares productivas (UFP), y en promedio el nivel de vida es bajo. Por otro lado, la digitalización de variables e índices por medio de coordenadas geográficas, ayudan a la correspondencia de comunidades rurales.

La población muestreada en las comunidades rurales tuvo un comportamiento progresivo, en donde más del $78 \%$ de la población es menor de 40 años, y la tasa promedio de analfabetismo en las comunidades fue del $11 \%$.

Las variables discriminantes en primer grado fueron la religión, la tenencia de tierra, el ICVV y el sexo del informante. Dichas variables, separaron las comunidades del municipio de Las Sabanas y las comunidades del municipio de Nandaime.

\section{REFERENCIAS BIBLIOGRÁFICAS}

aepcfa-girona, 2013. Año lectivo de 2013: Nicaragua consolida el camino hacia la verdadera erradicación del analfabetismo. Información sobre la alfabetización en Nicaragua. http://aepcfagirona.blogspot.com/2013/01/segunradio-la-primerisima-durante-2012_11.html.

Aguilar-Barojas S. 2005. Fórmulas para el cálculo de la muestra en investigaciones de salud. Salud en Tabasco. Vol. 11. Núm. 1-2, eneroagosto. Secretaria de Salud del Estado de Tabasco. México. p. 333-338.

ArcGIS. 2010. [software GIS]. Versión 10.0. Redlands, CA: Environmental Systems Research Institute, Inc.

Balzarini MG; González L; Tablada M; Casanoves F; Di Rienzo JA; Robledo CW. (2008). Infostat. Manual del Usuario, Editorial Brujas, Córdoba, Argentina.

CELADE. 2004. Boletín demográfico. Demographic Bulletin. América Latina y Caribe: Estimaciones y Proyecciones de Población 19502050. Latin America and Caribbean. Population Estimates and Projections. Año/Year XXXVII, No. 73. Centro Latinoamericano y Caribeño de Demografia (CELADE) - División de Población Latin American and Caribbean Demographic Centre (CELADE) - Population Division. Santiago de Chile / Santiago, Chile. 252 p.

CCPDH (Consejo Centroamericano de Procuradores de Derechos Humanos). 2008. Políticas públicas regionales sobre la reducción de la pobreza en Centroamérica y su incidencia en el pleno disfrute de los derechos humanos. Informe nacional Nicaragua. (en línea). Disponible en http://www.iidh.ed.cr/BibliotecaWeb/Varios/Documentos/BD_125911109/politicas_reduccion_pobreza_nicaragua.pdf.

COVEG. 2013. Indicador 3. Índice de masculinidad. 3 p. https: //www.coveg.gob.mx/seiisv/modulos/.../Indicador\%203.pdf.

Cuadras, MC. 2012. Nuevos métodos de análisis multivariante. CMC Editions, Manacor 30. Barcelona, Spain. 304 p. http://www.ub.edu/stat/ personal/cuadras/metodos.pdf.

Di Rienzo JA; Casanoves, F; Balzarini, MG; González, L; Tablada, M; Robledo, CW. 2012. InfoStat versión 2012. Grupo InfoStat, FCA, Universidad Nacional de Córdoba, Argentina. URL http://www.infostat.com.ar.

DGCyE (Dirección de Información y Estadística). 2005. Indicadores educativos, comparación de 13 países latinoamericanos y provincia de Buenos Aires. Dirección Provincial de Planteamiento. 14 p.

FIDEG. 2009. Encuesta de hogares para la medición de la Pobreza en Nicaragua. 30 p. 1283290135_Resultados FIDEG 2009web. (en línea). Disponible en http://hdr.undp.org/en/media/HDR_20072008_SP_Complete.pdf. / http://www.bio-nica.info/Biblioteca/INIDE2007ProyeccionPoblacional.pdf.

IBM SPSS Statistics 19 Command, Syntax Reference. Copyright (C) SPSS Inc. 1989, 2010. IBM SPSS Statistics version 19. 2483 p.

INEC (Instituto Nacional de Estadísticas y Censos, NI). 1995. http://www.inide.gob.ni/censo95/censo95.htm.

INIDE (Instituto Nacional de Información de Desarrollo). 2007. Nicaragua: Estimaciones y proyecciones de población nacional. $1950-2050$. Revisión 2007. 39 p. 
Instituto Nacional para la Evaluación de la Educación (INEE). 2008. Porcentaje de población analfabeta (2008). 8 p. http://www.inee.edu.mx/ bie/mapa_indica/2010/Panorama EducativoDeMexico/CS/CS03/2010_CS03 c-vinculo.pdf.

Ledesman, RG, Molina IP; Valero, M. 2002. Análisis de consistencia interna mediante Alfa de Cronbach: un programa basado en gráficos diná micos. Mar de Plata, Argentina. Psico-USF, vol. 7, p. 143-152.

Lestage, A. 1982. Analfabetismo y alfabetización. Organización de las Naciones Unidas para la Educación, la Ciencia y la Cultura (UNESCO). $32 \mathrm{p}$.

MECD (Ministerio de educación, cultura y deportes, NI). 2004. El desarrollo de la educación. Informe Nacional de Nicaragua. Agosto 2004. 37 p. http://www.ibe.unesco.org/International/ICE47/English/Natreps/reports/nicaragua_part_1.pdf.

Pla, EL; 1986. Análisis multivariado: método de componentes principales. Monografía 27. Serie de Matemática. Secretaría General de la Orga nización de la OEA, programa regional de desarrollo científico y tecnológico. Washington, D.C.

PNUD (Programa de la Naciones Unidas para el Desarrollo). 2007. Informe sobre desarrollo humano 2007-2008. La lucha contra el cambio climático: Solidaridad frente a un mundo dividido. p. 231-280.

Querol, D. Benavides A. Cisne C, J. 2013. Guía técnica 01. Integración de la UNA en el desarrollo rural de Nicaragua. Programa de Desarrollo Participativo Integral Rural (DEPARTIR). Universidad Nacional Agraria. 26 p.

Raimundini SL., M. Bianchi, N. A. Santos, L. P. Lopes Fávero, P. Schmidt, 2009. Percepções sobre o ensino da contabilidade introdutória para não contadores: A perspectiva dos discentes das universidades federais do estado do Rio Grande do Sul. Brasil. Revista de Edução e Pesquisa em Contabilidade (REPEC), v. 3, No. 3, art. 5 p. 85-105.

Salas-Bourgoin MA. 2012. Propuesta de índice de calidad de vida en la vivienda. Cuadernos CENDES, vol. 29. Núm. 79, enero-abril, 2012. Universidad Central de Venezuela. p. 57-58.

UCA (Universidad Centroamericana, NI). 2010. Problemática de la pobreza en Nicaragua. (en línea). Disponible en http://aulaweb.uca.edu.ni/ blogs/edlacayo/files/2010/08/Pobreza-de-Nic.pdf.

URL http://unesdoc.unesco.org/images/0013/001339/133942so.pdf. 